\title{
PRODUCTS IN HIGHER CHOW GROUPS AND MOTIVIC COHOMOLOGY
}

\author{
Charles Weibel
}

\begin{abstract}
We prove a compatibility theorem for the various products which have been defined on several cohomology theories: Bloch's higher Chow groups $C H^{i}(X, n)$ [B1]; Bivariant cycle cohomology $A_{r, n}(S, X)$ [FV]; Voevodsky's motivic cohomology $H_{\mathcal{M}}^{j}(X ; \mathcal{Z}(i))[\mathrm{V} 1]$; and étale cohomology (for finite coefficients).
\end{abstract}

Recently, several cycle cohomology groups have been introduced for schemes of finite type over a field $k$. These include the higher Chow groups $C H^{i}(X, n)$ of Bloch [B1], the bivariant cycle cohomology groups $A_{r n}(X)=A_{r n}(k, X)$ of Friedlander and Voevodsky $[\mathrm{FV}]$, and the motivic cohomology groups $H_{\mathcal{M}}^{m}(X, \mathbb{Z}(i))$ of Voevodsky [V1]. In reasonable situations, such as when $X$ is smooth and the field $k$ admits resolution of singularities (e.g., $\operatorname{char}(k)=0$ ), we know by [FV], [S2] and [V1] that these groups are isomorphic: if we set $m+n=2 i$ and $r+i=\operatorname{dim}(X)$ then

$$
C H^{i}(X, n) \cong A_{r n}(X) \cong H_{\mathcal{M}}^{m}(X, \mathbb{Z}(i)) .
$$

The main result in this paper is that these isomorphisms identify the various cohomology products which have been defined in [B1], [FV] and [V1] respectively.

Main Theorem 0.2. Let $X$ be smooth over a field $k$ which admits resolution of singularities. Then the isomorphism of (0.1) identifies the product on Bloch's higher Chow groups, the product on bivariant cycle cohomology, and the product on motivic cohomology.

That is, we shall identify Bloch's product [B1, 5.7]

$$
C H^{i}(X, m) \otimes C H^{j}(X, n) \rightarrow C H^{i+j}(X, m+n),
$$

the Friedlander-Voevodsky product $[\mathrm{FV}, 8.6]$

$$
A_{r m}(X) \otimes A_{s n}(X) \rightarrow A_{r+s-d, m+n}(X), \quad d=\operatorname{dim}(X),
$$

and Voevodsky's product in motivic cohomology [V1]

$$
H_{\mathcal{M}}^{m}(X, \mathbb{Z}(i)) \otimes H_{\mathcal{M}}^{n}(X, \mathbb{Z}(j)) \rightarrow H_{\mathcal{M}}^{m+n}(X, \mathbb{Z}(i+j)) .
$$

We will also show that the natural map from motivic cohomology with finite coefficients to étale cohomology $[\mathrm{SV}]$ is also compatible with products. 
In the first two sections, we give a simplicial description of Bloch's product (0.3), and interpret this in terms of equidimensional cycles. In section 3 we construct an external pairing for bivariant cycle cohomology groups $A_{r n}(S, X)$. Via the pullback, this gives an internal product on the groups $A_{r n}(S, X)$, and generalizes the pairing (0.4) defined in [FV]. When $k$ admits resolution of singularities and $X$ is smooth, we show that this agrees with Bloch's product under the isomorphism $C H^{i}(X, n) \cong$ $A_{r n}(\operatorname{Spec} k, X)$ of $[\mathrm{FV}, 8.1]$, where $i=\operatorname{dim}(X)-r$.

In section 4 we consider the motivic cohomology groups $H_{\mathcal{M}}^{m}(X ; \mathbb{Z}(i))$. An argument, due to Voevodsky, shows that the isomorphism between $A_{r n}(X)=$ $A_{r n}(\operatorname{Spec} k, X)$ and $H_{\mathcal{M}}^{2 i-n}(X ; \mathbb{Z}(i))$ identifies the corresponding cohomology products. In section 5 we show that the natural map from $H_{\mathcal{M}}^{2 i-n}\left(X ; \mathbb{Z} / \ell^{\nu}(i)\right)$ to étale cohomology is compatible with products. When $\ell=2$, Voevodsky has proven in [V2] that that this map is an isomorphism.

This paper was originally intended to be a part of the paper [RW]. I am grateful to John Rognes for his careful reading of an earlier version of this material, and his permission to extract it into this paper. I would also like to thank V. Voevodsky for several discussions about motivic cohomology, including his napkin sketch of Proposition 4.5.

\section{The product on Bloch's higher Chow groups}

We begin by recalling the definition of Bloch's higher Chow groups from [B1]. We shall then reinterpret the product, which is also defined in [B1], in order to introduce our simplicial point of view. Recall that there is a cosimplicial scheme $\Delta$, where $\Delta^{p}=\operatorname{Spec}\left(k\left[t_{0}, \ldots, t_{p}\right] /\left(\sum t_{j}=1\right)\right)$. The cosimplicial structure allows us to talk about faces of $\Delta^{p}$ or even $X \times \Delta^{p}$.

Given a quasi-projective scheme $X$ over $k$ and a positive integer $i$, Bloch defines $C H^{i}(X, p)$ to be the $p$ th homotopy group of the simplicial abelian group $p \mapsto$ $\mathcal{Z}_{\mathrm{Bl}}^{i}(X, p)$, where $\mathcal{Z}_{\mathrm{Bl}}^{i}(X, p)$ is the free abelian group on the irreducible subvarieties of codimension $i$ in $X \times \Delta^{p}$ which meet all faces properly (i.e., in a subscheme of codimension $\geq i)$. By the Dold-Kan correspondence, $C H^{i}(X, p)$ is also the $p$ th homology of the chain complex $C \mathcal{Z}_{\mathrm{Bl}}^{i}(X)$ associated to $\mathcal{Z}_{\mathrm{Bl}}^{i}(X, \cdot)$.

Recall that if $A$.. is a bisimplicial abelian group, its total complex Tot $A$ is the total complex of the associated double chain complex. Its diagonal $\operatorname{diag} A$ is the simplicial abelian group $p \mapsto A_{p p}$ and we write $C \operatorname{diag} A$ for the associated chain complex. By the Eilenberg-Zilber Theorem [W2, 8.5.1], the homology groups of $C \operatorname{diag} A$ are the same as the homology groups of Tot $A$, either by the Alexander-Whitney map or its inverse $\nabla_{*}$ : Tot $A \rightarrow C \operatorname{diag}(A)$. The component $A_{p q} \rightarrow A_{p+q, p+q}$ of $\nabla_{*}$ is the sum over all $(p, q)$-shuffles $\mu$ of $(-1)^{\mu}$ times the map induced by a degeneracy $\sigma(\mu): \Delta^{p+q} \times \Delta^{p+q} \rightarrow \Delta^{p} \times \Delta^{q} ;$ see [W2, p. 278].

Combinatorially, it is well known that the set of all $(p, q)$-shuffles $\mu$ indexes the $(p+q)$-simplices $\theta_{\mu}: \Delta^{p+q} \rightarrow \Delta^{p} \times \Delta^{q}$ in a simplicial subdivision of the polyhedron $\left|\Delta^{p}\right| \times\left|\Delta^{q}\right|$. Geometrically, the map $\theta_{\mu}$ is the composition of $\sigma(\mu)$ with the diagonal $\Delta^{p+q} \hookrightarrow \Delta^{p+q} \times \Delta^{p+q}$; as a scheme map, each $\theta_{\mu}$ is an isomorphism. By the faces of $X \times \Delta^{p} \times \Delta^{q}$ we shall mean the faces under this simplicial subdivision; each face of $X \times \Delta^{p} \times \Delta^{q}$ arises from a face of $\Delta^{p+q}$ under some isomorphism $\theta_{\mu}$. It follows that the collection $\left\{\theta_{\mu}\right\}$ forms a triangulation in the sense of $[B 1$, p. 282]. 
Let $\mathcal{Z}_{\mathrm{Bl}}^{i}(X ; p, q)$ denote the bisimplicial abelian group which is free in bidegree $(p, q)$ on the irreducible subvarieties of $X \times \Delta^{p} \times \Delta^{q}$ meeting all faces properly. The maps $\mathcal{Z}_{\mathrm{Bl}}^{i}(X ; p, p) \rightarrow \mathcal{Z}_{\mathrm{Bl}}^{i}(X, p)$, defined by intersection with the diagonal face $\Delta^{p} \subset \Delta^{p} \times \Delta^{p}$, assemble to form a simplicial map $\operatorname{diag} \mathcal{Z}_{\mathrm{Bl}}^{i}(X ; \cdot, \cdot) \stackrel{\delta}{\rightarrow} \mathcal{Z}_{\mathrm{Bl}}^{i}(X, \cdot)$. Geometrically, if $\mu$ is a $(p, q)$-shuffle then the composition of $\sigma(\mu)^{*}: \mathcal{Z}_{\mathrm{Bl}}^{i}(X ; p, q) \rightarrow$ $\mathcal{Z}_{\mathrm{Bl}}^{i}(X ; p+q, p+q)$ with $\delta$ is the map $\mathcal{Z}_{\mathrm{Bl}}^{i}(X ; p, q) \rightarrow \mathcal{Z}_{\mathrm{Bl}}^{i}(X, p+q)$ induced by $\theta_{\mu}: \Delta^{p+q} \rightarrow \Delta^{p} \times \Delta^{q}$. This yields a simple formula, which we record:

Lemma 1.1. The $(p, q)$ component of the chain map $\operatorname{Tot} \mathcal{Z}_{\mathrm{Bl}}^{i}(X ; \cdot, \cdot) \stackrel{\delta \nabla_{*}}{\longrightarrow} C \mathcal{Z}_{\mathrm{Bl}}^{i}(X)$ satisfies:

$$
\delta \nabla_{*}=\sum(-1)^{\mu} \delta \sigma(\mu)^{*}=\sum(-1)^{\mu} \theta_{\mu}^{*} .
$$

Following Bloch, define $\mathcal{Z}_{\mathrm{Bl}}^{i+j}(X, Y ; \cdot, \cdot)^{\prime}$ to be the bisimplicial abelian subgroup of $\mathcal{Z}_{\mathrm{Bl}}^{i}(X, \cdot) \otimes \mathcal{Z}_{\mathrm{Bl}}^{j}(Y, \cdot)$ generated by products $Z \otimes W$, where $Z \subset X \times \Delta^{p}$ and $W \subset Y \times \Delta^{q}$ are such that $Z \times W$ meets all faces of $X \times Y \times \Delta^{p} \times \Delta^{q}$ properly. By construction, there is a bisimplicial map from $\mathcal{Z}_{\mathrm{Bl}}^{i+j}(X, Y ; \cdot)^{\prime}$ to $\mathcal{Z}_{\mathrm{Bl}}^{i+j}(X \times Y ; \cdot, \cdot)$, sending $Z \otimes W$ to $Z \times W$. Bloch proves in [B1, 5.1] that the inclusion

$$
\mathcal{Z}_{\mathrm{Bl}}^{i+j}(X, Y ; \cdot)^{\prime} \subset \mathcal{Z}_{\mathrm{Bl}}^{i}(X, \cdot) \otimes \mathcal{Z}_{\mathrm{Bl}}^{j}(Y, \cdot)
$$

is a quasi-isomorphism. If we compose with the map $\delta \nabla_{*}$

$$
\begin{aligned}
\operatorname{Tot} \mathcal{Z}_{\mathrm{Bl}}^{i}(X, \cdot) \otimes \mathcal{Z}_{\mathrm{Bl}}^{j}(Y, \cdot) & \stackrel{\sim}{\operatorname{Tot}} \mathcal{Z}_{\mathrm{Bl}}^{i+j}(X, Y ; \cdot)^{\prime} \rightarrow \operatorname{Tot} \mathcal{Z}_{\mathrm{Bl}}^{i+j}(X \times Y ; \cdot, \cdot) \\
& \stackrel{\nabla_{*}}{\longrightarrow} C \operatorname{diag} \mathcal{Z}_{\mathrm{Bl}}^{i+j}(X \times Y ; \cdot, \cdot) \stackrel{\delta}{\longrightarrow} C \mathcal{Z}_{\mathrm{Bl}}^{i+j}(X \times Y, \cdot)
\end{aligned}
$$

and use the Künneth formula, we obtain a pairing of bigraded abelian groups

$$
C H^{i}(X, m) \otimes C H^{j}(Y, n) \rightarrow C H^{i+j}(X \times Y, m+n) .
$$

Lemma 1.3. The pairing (1.2) agrees with the external product defined in [B1] on the higher Chow groups $C H^{i}(X, \cdot)$.

Proof. Given Lemma 1.1, this follows from the definition of Bloch's product on p. 282 of [B1].

\section{EQUIDIMENSIONAL CYCLES}

Our second task is to interpret Bloch's external product in terms of equidimensional cycles. A morphism $X \rightarrow Y$ of schemes is called equidimensional of relative dimension $r$ if it is dominant, of finite type, and each fiber has pure dimension $r$. The following definition is from $[\mathrm{SV} 2,3.1]$, although the notation is taken from $[\mathrm{FV}]$.

Definition 2.1. Let $X$ be quasiprojective and $r \geq 0$. For each smooth $U$, we write $\mathcal{Z}_{\text {equi }}(X, r)(U)$ for the free abelian group on the integral subschemes $Z$ of $X \times U$ which are equidimensional of relative dimension $r$ over $U$.

It is known [SV2, 3.3.15 and 3.6.3] that $\mathcal{Z}_{\text {equi }}(X, r)(U)$ is contravariant and functorial in $U$, i.e., it is a presheaf on the smooth Zariski site $S m / k$. Replacing $U$ by the cosimplicial scheme $\Delta^{\cdot}$ yields the simplicial abelian group $\mathcal{Z}_{\text {equi }}(X, r)\left(\Delta^{*}\right)$. 
We shall assume that $0 \leq r \leq \operatorname{dim}(X)$, since $\mathcal{Z}_{\text {equi }}(X, r)$ is zero otherwise. When $X$ is equidimensional, it is notationally convenient to set $i=\operatorname{dim}(X)-r$, so $0 \leq i \leq \operatorname{dim}(X)$, and write $\mathcal{Z}_{\text {equi }}^{i}(X, \cdot)$ for the $\operatorname{simplicial~group~} \mathcal{Z}_{\text {equi }}(X, r)\left(\Delta^{*}\right)$.

A subscheme $Z \subset X \times \Delta^{n}$ which is equidimensional over $\Delta^{n}$ meets every subscheme of the form $X \times W$ properly. Hence $\mathcal{Z}_{\text {equi }}^{i}(X, \cdot)$ is a simplicial subgroup of $\mathcal{Z}_{\mathrm{Bl}}^{i}(X, \cdot)$. Suslin proved that this inclusion is a homotopy equivalence:

Theorem 2.2. (Suslin) Let $X$ be an equidimensional scheme of finite type over $k$, and suppose that $0 \leq i \leq \operatorname{dim}(X)$. If $X$ is affine, or if $k$ admits resolution of singularities, then for all $n$ :

$$
C H^{i}(X, n) \cong \pi_{n} \mathcal{Z}_{\text {equi }}^{i}(X, \cdot)
$$

Proof. Suslin proved the result in the affine case [S2, 2.1]. The extension using resolution of singularities follows from [FV, 5.11] and [B2], or from the second to last paragraph in the proof of $[\mathrm{V} 1,4.2 .9]$.

Let $C \mathcal{Z}_{\text {equi }}^{i}(X)$ denote the chain complex associated to $\mathcal{Z}_{\text {equi }}^{i}(X, \cdot)$.

Lemma 2.3. If $0 \leq i \leq \operatorname{dim}(X)$ and $0 \leq j \leq \operatorname{dim}(Y)$, there is a natural pairing

$$
\text { Tot } \mathcal{Z}_{\text {equi }}^{i}(X, \cdot) \otimes \mathcal{Z}_{\text {equi }}^{j}(Y, \cdot) \rightarrow C \mathcal{Z}_{\text {equi }}^{i+j}(X \times Y)
$$

If $Z \in \mathcal{Z}_{\text {equi }}^{i}(X, p)$ and $Z^{\prime} \in \mathcal{Z}_{\text {equi }}^{j}(Y, q)$, this pairing sends $Z \otimes Z^{\prime}$ to the alternating sum $\sum(-1)^{\mu} \theta_{\mu}^{*}\left(Z \times Z^{\prime}\right)$, taken over all $(p, q)$-shuffles $\mu$, of the pullbacks of the cycle $Z \times Z^{\prime}$ along the isomorphisms $\theta_{\mu}: \Delta^{p+q} \cong \Delta^{p} \times \Delta^{q}$.

Proof. If $Z \subset X \times \Delta^{p}$ and $Z^{\prime} \subset Y \times \Delta^{q}$ are equidimensional of relative dimensions $r=\operatorname{dim}(X)-i$ and $s=\operatorname{dim}(Y)-j$ over $\Delta^{p}$ and $\Delta^{q}$ respectively, then the composition $Z \times Z^{\prime} \rightarrow Z \times \Delta^{q} \rightarrow \Delta^{p} \times \Delta^{q}$ is equidimensional of relative dimension $r+s$. Hence $Z \times Z^{\prime}$ belongs to the subgroup $\mathcal{Z}_{\text {equi }}^{i+j}(X \times Y ; p, q)$ of $\mathcal{Z}_{\mathrm{Bl}}^{i+j}(X \times Y ; p, q)$, defining a bisimplicial map from $\mathcal{Z}_{\text {equi }}^{i}(X, \cdot) \otimes \mathcal{Z}_{\text {equi }}^{j}(Y, \cdot)$ to $\mathcal{Z}_{\text {equi }}^{i+j}(X \times Y ; \cdot, \cdot)$. As in $\S 1$, the product is obtained by composing with $\delta \nabla_{*}$ and using the Künneth formula.

Corollary 2.4. If $0 \leq i \leq \operatorname{dim}(X)$ and $0 \leq j \leq \operatorname{dim}(Y)$, the induced product on higher Chow groups

$$
C H^{i}(X, m) \otimes C H^{j}(Y, n) \rightarrow C H^{i+j}(X \times Y, m+n)
$$

may be identified with Bloch's external product (1.2).

Proof. Since the equidimensional $Z \times Z^{\prime}$ meets every subvariety properly, the tensor product $\mathcal{Z}_{\text {equi }}^{i}(X, p) \otimes \mathcal{Z}_{\text {equi }}^{j}(Y, q)$ is a subgroup of $\mathcal{Z}_{\text {equi }}^{i+j}(X, Y ; p, q)^{\prime}$, and $\mathcal{Z}_{\text {equi }}^{i}(X, \cdot) \otimes \mathcal{Z}_{\text {equi }}^{j}(Y, \cdot)$ is a bisimplicial subgroup of $\mathcal{Z}_{\mathrm{Bl}}^{i+j}(X, Y ; \cdot, \cdot)^{\prime}$. This establishes commutativity of the diagram (of chain complexes):

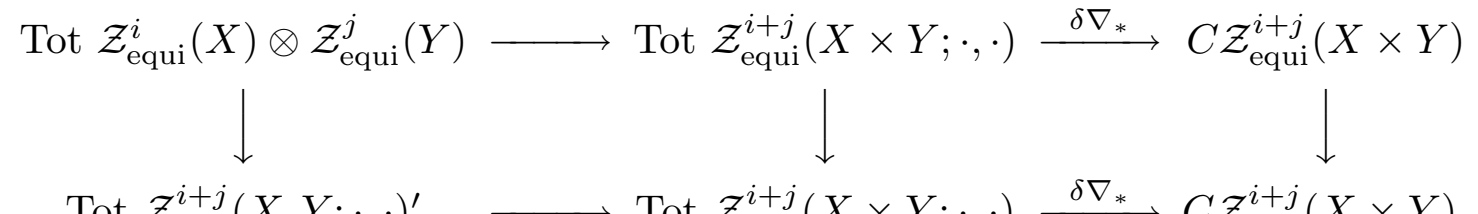

The result now follows from Lemma 1.3, since the bottom composite is (1.2). 
Remark 2.5. To take care of the case when $i>\operatorname{dim}(X)$ or $j>\operatorname{dim}(Y)$, we may replace $X$ and $Y$ in Corollary 2.4 by $X \times \mathbb{A}^{i}$ and $Y \times \mathbb{A}^{j}$, respectively. This works because Bloch's product is natural and hence compatible with the isomorphisms $C H^{i}(X, n) \cong C H^{i}\left(X \times \mathbb{A}^{i}, n\right)$, etc.

\section{Products in BIVARIANT CYCLE COHOMOLOGY}

Recall from $[\mathrm{FV}, 4.3]$ that the bivariant cycle groups $A_{r n}(S, X)$ of a smooth scheme $S$ and a quasiprojective scheme $X$ are defined as the hypercohomology groups $H_{c d h}^{-n}\left(S, \underline{C}_{*} \mathcal{Z}_{\text {equi }}(X, r)\right)$ for the smooth $c d h$ site of $S$. Here $\underline{C}_{*} \mathcal{Z}_{\text {equi }}(X, r)$ denotes the chain complex (of presheaves) associated to the simplicial presheaf $U \mapsto$ $\mathcal{Z}_{\text {equi }}(X, r)\left(U \times \Delta^{\cdot}\right)$, but of course it must be sheafified before taking cohomology.

We shall suppose that $k$ admits resolution of singularities; this is certainly true if $k$ has characteristic 0 . By [FV, 8.1] we have the simpler formula

$$
A_{r n}(S, X)=H_{n} \underline{C}_{*} \mathcal{Z}_{\text {equi }}(X, r)(S)=\pi_{n} \mathcal{Z}_{\text {equi }}(X, r)\left(S \times \Delta^{\cdot}\right), \quad r \geq 0 .
$$

In particular, if $S=\operatorname{Spec} k$ and $i=\operatorname{dim}(X)-r$ then using Theorem 2.2 we have

$$
C H^{i}(X, n)=\pi_{n} \mathcal{Z}_{\text {equi }}^{i}(X, \cdot) \cong A_{r n}(\operatorname{Spec} k, X), \quad 0 \leq i \leq \operatorname{dim}(X) .
$$

If $i>\operatorname{dim}(X)$, we can cross with the affine space $\mathbb{A}^{i}$ and use homotopy invariance of Bloch's higher Chow groups, $C H^{i}(X, n) \cong C H^{i}\left(X \times \mathbb{A}^{i}, n\right)$. This yields the following result; cf. [FV, 8.3(2)].

Proposition 3.1. Suppose that $k$ admits resolution of singularities, and that $X$ is quasiprojective of dimension $d=\operatorname{dim}(X)$. Then for all $i \geq 0$

$$
C H^{i}(X, n) \cong C H^{i}\left(X \times \mathbb{A}^{i}, n\right) \cong A_{d n}\left(\operatorname{Spec} k, X \times \mathbb{A}^{i}\right) .
$$

Lemma 3.2. Suppose that $k$ admits resolution of singularities, and that $X$ and $Y$ are quasiprojective over $k$, of dimensions $d=\operatorname{dim}(X)$ and $e=\operatorname{dim}(Y)$. Then for each smooth $S$ there is an external pairing

$$
A_{r m}(S, X) \otimes A_{s n}(S, Y) \rightarrow A_{r+s, m+n}(S, X \times Y), \quad(0 \leq r \leq d, 0 \leq s \leq e) .
$$

If $S=$ Spec $k$, Bloch's external product (1.2) coincides with this external pairing in this range. Outside this range, Bloch's product (1.2) coincides with the pairing

$$
A_{d m}\left(S, X \times \mathbb{A}^{i}\right) \otimes A_{e n}\left(S, Y \times \mathbb{A}^{j}\right) \rightarrow A_{d+e, m+n}\left(S, X \times Y \times \mathbb{A}^{i+j}\right) .
$$

Proof. Fix $r, s \geq 0$. Following section 8 of $[\mathrm{FV}]$, there is a pairing of presheaves

$$
\mathcal{Z}_{\text {equi }}(X, r) \otimes \mathcal{Z}_{\text {equi }}(Y, s) \stackrel{\times}{\longrightarrow} \mathcal{Z}_{\text {equi }}(X \times Y, r+s),
$$

sending $Z \otimes W$ to the cycle associated to the subscheme $Z \times_{U} W$ of $X \times Y \times U$. Applying this pairing to $U=S \times \Delta^{\cdot}$ yields a simplicial map

$$
\operatorname{diag} \mathcal{Z}_{\text {equi }}(X, r)\left(S \times \Delta^{*}\right) \otimes \mathcal{Z}_{\text {equi }}(Y, s)\left(S \times \Delta^{*}\right) \stackrel{\times}{\longrightarrow} \mathcal{Z}_{\text {equi }}(X \times Y, r+s)\left(S \times \Delta^{*}\right) \text {. }
$$

Applying the Eilenberg-Zilber Theorem [W2, 8.5.3], this yields a morphism of chain complexes of abelian groups for each $S$ :

(3.2.2) Tot $\underline{C}_{*} \mathcal{Z}_{\text {equi }}(X, r)(S) \otimes \underline{C}_{*} \mathcal{Z}_{\text {equi }}(Y, s)(S) \stackrel{\times \nabla_{*}}{\longrightarrow} \underline{C}_{*} \mathcal{Z}_{\text {equi }}(X \times Y, r+s)(S)$.

The pairing is now immediate from the Künneth Formula. When $S=\operatorname{Spec} k$, the map $\times \nabla_{*}$ in (3.2.2) becomes the pairing of Lemma 2.3, so the agreement with $(1.2)$ follows from Corollary 2.4, Prop. 3.1 and Remark 2.5. 
Remark 3.3. Without assuming resolution of singularities, we could construct the external pairing as follows. First we interpret (3.2.2) as a functor of $S$, i.e., as a morphism of presheaves. Then we modify Jardine's argument in [J, p.208]: fix $S$ and a pairing of simplicial sheaves $A \otimes B \rightarrow C$. For each trivial fibration $T \rightarrow S$, we get a cochain map $\operatorname{hom}(\mathbb{Z} T, A) \otimes \operatorname{hom}(\mathbb{Z} T, B) \rightarrow \operatorname{hom}(\mathbb{Z} T, C)$ in the usual way; the desired product comes from the colimit of the induced pairings over all $T$.

Corollary 3.4. Assume $k$ admits resolution of singularities. Then the the isomorphisms $A_{r n}(S, Y) \cong A_{r+1, n}\left(S, Y \times \mathbb{A}^{1}\right)$ of $[\mathrm{FV}, 8.3]$, identify the external product of Lemma 3.2 with the external product

$$
A_{r m}(S, X) \otimes A_{s+1, n}\left(S, Y \times \mathbb{A}^{1}\right) \rightarrow A_{r+s+1, m+n}\left(S, X \times Y \times \mathbb{A}^{1}\right) .
$$

To get an internal pairing, we need pullback maps for bivariant cycle cohomology. Our use of $[\mathrm{FV}]$ requires that $X$ be smooth and that $k$ admits resolution of singularities.

Definition 3.5. Suppose that $f: X \rightarrow Y$ is a morphism of smooth schemes, and set $d=\operatorname{dim}(X), e=\operatorname{dim}(Y)$. We define the "pullback" morphism $A_{t, n}(S, Y) \rightarrow$ $A_{t+d-e, n}(S, X)$ to be the following composite, obtained using homotopy invariance in $S[\mathrm{FV}, 5.9]$ and duality for $X$ and $Y[\mathrm{FV}, 8.2]$.

$$
\begin{aligned}
A_{t n}(S, Y) \cong A_{t n}\left(S \times \mathbb{A}^{e}, Y\right) & \stackrel{\sim}{\longrightarrow} A_{t+e, n}\left(S, \mathbb{A}^{e} \times Y\right) \\
& \stackrel{\sim}{\sim} A_{t n}\left(S \times Y, \mathbb{A}^{e}\right) \stackrel{f^{*}}{\longrightarrow} A_{t n}\left(S \times X, \mathbb{A}^{e}\right) \\
& \stackrel{\sim}{\longrightarrow} A_{t+d, n}\left(S, \mathbb{A}^{e} \times X\right) \stackrel{\sim}{\longleftarrow} A_{t+d-e, n}\left(S \times \mathbb{A}^{e}, X\right) \cong A_{t+d-e, n}(S, X) .
\end{aligned}
$$

To simplify notation even further, we will write $A_{r n}(X)$ for $A_{r n}(\operatorname{Spec} k, X)$. Recall that we are assuming that $k$ admits resolution of singularities.

Proposition 3.6. Let $f: X \rightarrow Y$ be a morphism of smooth schemes over $k$. Then the pullback morphism $A_{t, n}(Y) \rightarrow A_{t+d-e, n}(X)$ is the same as the pullback map $f^{*}: C H^{i}(Y, n) \rightarrow C H^{i}(X, n)$ defined by Bloch in $[\mathrm{B} 1,4.1]$, where $i=\operatorname{dim}(Y)-t$.

Proof. It suffices to show that the map $A_{t+e, n}\left(\mathbb{A}^{e} \times Y\right) \rightarrow A_{t+d, n}\left(\mathbb{A}^{e} \times X\right)$ agrees with Bloch's pullback $C H^{i}\left(\mathbb{A}^{e} \times Y, n\right) \rightarrow C H^{i}\left(\mathbb{A}^{e} \times X, n\right)$. By $[\mathrm{FV}, 7.1]$ the simplicial inclusion

$$
\mathcal{Z}_{\text {equi }}\left(\mathbb{A}^{e}, t\right)\left(Y \times \Delta^{\cdot}\right) \hookrightarrow \mathcal{Z}_{\text {equi }}\left(\mathbb{A}^{e} \times Y, t+e\right)\left(\Delta^{\cdot}\right)
$$

is a homotopy equivalence. From [FV, 8.1] we see that this induces the duality isomorphism $A_{t n}\left(Y, \mathbb{A}^{e}\right) \cong A_{t+e, n}\left(\operatorname{Spec} k, \mathbb{A}^{e} \times Y\right)$ of $[\mathrm{FV}, 8.2]$. On the other hand, $\mathcal{Z}_{\text {equi }}\left(\mathbb{A}^{e}, t\right)\left(Y \times \Delta^{\cdot}\right)$ is contained in the subcomplex $\mathcal{Z}_{f}^{i}\left(\mathbb{A}^{e} \times Y, \cdot\right)$ of $\mathcal{Z}_{\mathrm{Bl}}^{i}\left(\mathbb{A}^{e} \times Y, \cdot\right)$ used by Bloch in $[\mathrm{B} 1,4.1]$ to define the map $f^{*}: C H^{i}(Y, n) \rightarrow C H^{i}(X, n)$. Indeed, if $Y^{\prime}$ is a closed subvariety of $Y$ then any $Z \subset \mathbb{A}^{e} \times Y \times \Delta^{n}$ which is equidimensional over $Y \times \Delta^{n}$ meets $\mathbb{A}^{e} \times Y^{\prime} \times \Delta^{n}$ properly. It follows that Bloch's product is defined by the same pullback map

$$
f^{*}: \mathcal{Z}_{\text {equi }}\left(\mathbb{A}^{e}, t\right)\left(Y \times \Delta^{*}\right) \rightarrow \mathcal{Z}_{\text {equi }}\left(\mathbb{A}^{e}, t\right)\left(X \times \Delta^{\cdot}\right) \stackrel{\sim}{\longrightarrow} \mathcal{Z}_{\text {equi }}\left(\mathbb{A}^{e} \times X, t+e\right)\left(\Delta^{*}\right)
$$

used to define the pullback map on bivariant cycle cohomology. 
Definition 3.7. If $X$ is smooth of dimension $d$ over a field $k$ admitting resolution of singularities, we define the internal product

$$
A_{r m}(S, X) \otimes A_{s n}(S, X) \rightarrow A_{r+s-d, m+n}(S, X)
$$

to be the composition of the external product (with $X=Y$ ) with the pullback map $A_{r+s, m+n}(S, X \times X) \rightarrow A_{r+s-d, m+n}(S, X)$ along the diagonal $X \hookrightarrow X \times X$.

When $S=$ Spec $k$, the internal product we have constructed coincides with the natural multiplication $A_{r m}(X) \otimes A_{s n}(X) \rightarrow A_{r+s-d, m+n}(X)$ defined by Friedlander and Voevodsky in $[\mathrm{FV}, 8.6]$. Indeed, their construction is a special case of the one we have given.

Combining 3.2 and 3.6 and setting $i=d-r, j=d-s$ we obtain the following comparison with Bloch's internal product (0.3), i.e., $C H^{i}(X, m) \otimes C H^{j}(X, n) \rightarrow$ $C H^{i+j}(X, m+n)$.

Corollary 3.8. Suppose $X$ is smooth of dimension $d$ over a field $k$ admitting resolution of singularities, and set $S=$ Spec $k$. If $0 \leq r, s \leq d$ and $r+s \geq d$, Bloch's internal product (0.3) agrees with the internal product

$$
A_{r m}(X) \otimes A_{s n}(X) \rightarrow A_{r+s-d, m+n}(X) .
$$

In general, Bloch's internal product (0.3) agrees with the internal product

$$
A_{d+j, m}\left(X \times \mathbb{A}^{i+j}\right) \otimes A_{d+i, n}\left(X \times \mathbb{A}^{i+j}\right) \rightarrow A_{d, m+n}\left(X \times \mathbb{A}^{i+j}\right)
$$

\section{Products in Motivic Cohomology}

Now we turn to the product in motivic cohomology, which comes from the tensor structure in the triangulated category $D M=D M_{-}^{\text {eff }}(k)$ of [V1]. Recall from [V1] that if $X$ is of finite type over a perfect field $k$ then the Suslin complex $\underline{C}_{*}(X)$ and its variant $\underline{C}_{*}^{c}(X)$ are complexes of sheaves in $D M$; formally $\underline{C}_{*}(X)(U)$ and $\underline{C}_{*}^{c}(X)(U)$ are the complexes associated to the simplicial sheaves $c_{\text {equi }}\left(U \times \Delta^{*}, X\right)$ and $\mathcal{Z}_{\text {equi }}(X, 0)\left(U \times \Delta^{*}\right)$, respectively. The category $D M$ also contains the complexes $\mathbb{Z}(i)$, which are determined by the formula $\mathbb{Z}(i)[2 i]=\underline{C}_{*}^{c}\left(\mathbb{A}^{i}\right)$ of $[\mathrm{V} 1,4.1 .8]$.

By definition, $H_{\mathcal{M}}^{m}(X, \mathbb{Z}(i))$ is $\operatorname{Hom}_{D M}\left(\underline{C}_{*}(X), \mathbb{Z}(i)[m]\right)$. We see by $[\mathrm{V} 1,4.2 .3]$, or by $[\mathrm{FV}, 9.2]$, that

$$
A_{0 n}\left(X, \mathbb{A}^{i}\right) \cong \operatorname{Hom}_{D M}\left(\underline{C}_{*}(X)[n], \mathbb{Z}(i)[2 i]\right) \cong H_{\mathcal{M}}^{2 i-n}(X, \mathbb{Z}(i)) .
$$

When $X$ is smooth, we have the following re-interpretation.

Proposition 4.2 (Suslin). Let $X$ be a smooth variety over a field $k$, which admits resolution of singularitites. If $d=\operatorname{dim}(X)$, there are natural isomorphisms

$$
C H^{i}(X, n) \cong A_{d n}\left(X \times \mathbb{A}^{i}\right) \cong H_{\mathcal{M}}^{2 i-n}(X ; \mathbb{Z}(i))
$$

Proof. By (4.1) and [FV 8.2], which requires $X$ to be smooth, we have:

$$
H_{\mathcal{M}}^{2 i-n}(X ; \mathbb{Z}(i)) \cong A_{0, n}\left(X, \mathbb{A}^{i}\right) \cong A_{d, n}\left(\operatorname{Spec} k, X \times \mathbb{A}^{i}\right)
$$

The result now follows from Proposition 3.1. 
Now the product $H_{\mathcal{M}}^{m}(X, \mathbb{Z}(i)) \otimes H_{\mathcal{M}}^{n}(X, \mathbb{Z}(j)) \rightarrow H_{\mathcal{M}}^{m+n}(X, \mathbb{Z}(i+j))$, mentioned in $(0.5)$, is obtained by applying $\operatorname{Hom}_{D M}\left(\underline{C}_{*}(X),-\right)$ to the "twisting" isomorphisms $\mathbb{Z}(i)[m] \otimes \mathbb{Z}(j)[n] \cong \mathbb{Z}(i+j)[m+n]$ in the tensor category $D M$. Tensoring the twisting isomorphisms with $\mathbb{Z} / \ell$ also yields products with coefficients $\bmod \ell$ :

$$
H_{\mathcal{M}}^{m}(X, \mathbb{Z} / \ell(i)) \otimes H_{\mathcal{M}}^{n}(X, \mathbb{Z} / \ell(j)) \rightarrow H_{\mathcal{M}}^{m+n}(X, \mathbb{Z} / \ell(i+j))
$$

Proposition 4.3. Let $S$ be smooth over a field $k$ admitting resolution of singularities. Then the motivic cohomology product (0.5) and the external product

$$
A_{0 m}\left(S, \mathbb{A}^{i}\right) \otimes A_{0 n}\left(S, \mathbb{A}^{j}\right) \rightarrow A_{0, m+n}\left(S, \mathbb{A}^{i+j}\right)
$$

of Lemma 3.2 are identified by the isomorphism (4.1).

Proof. Using $\underline{C}_{*}^{c}\left(\mathbb{A}^{i}\right) \cong \mathbb{Z}(i)[2 i]$, and setting $a=m-2 i, b=n-2 j$, the twisting isomorphisms may be rewritten as

$$
\underline{C}_{*}^{c}\left(\mathbb{A}^{i}\right)[a] \otimes \underline{C}_{*}^{c}\left(\mathbb{A}^{j}\right)[b] \stackrel{\cong}{\longrightarrow} \underline{C}_{*}^{c}\left(\mathbb{A}^{i+j}\right)[a+b]
$$

By [V1, 4.1.7], the twisting isomorphism (4.3.1) is induced by the FriedlanderVoevodsky pairing (3.2.1). Evaluating the presheaves at $S$ gives the pairing (3.2.2), and hence the external product of Lemma 3.2 for $A_{0 *}(S, X)$ with $X=\mathbb{A}^{i}$ and $Y=$ $\mathbb{A}^{j}$. Since evaluation of $(4.3 .1)$ at $S$ corresponds to applying $\operatorname{Hom}_{D M}\left(\underline{C}_{*}(S),-\right)$ by $[\mathrm{V} 1,4.2 .3]$, this proves the result.

Corollary 4.4. If $d=\operatorname{dim}(S)$, the motivic cohomology product (0.5) also coincides with the external product

$$
A_{r m}\left(S, \mathbb{A}^{d}\right) \otimes A_{s n}\left(S, \mathbb{A}^{d}\right) \rightarrow A_{r+s, m+n}\left(S, \mathbb{A}^{2 d}\right)
$$

Proof. Combine 4.3 with Corollary 3.4.

The following result is due to Voevodsky; his proof is more direct than this one, avoiding the external product.

Proposition 4.5. (Voevodsky) Assume $k$ admits resolution of singularities. If $X$ is smooth over $k$, and $r+i=\operatorname{dim}(X)$, the isomorphism $A_{r n}(X) \cong H_{\mathcal{M}}^{2 i-n}(X ; \mathbb{Z}(i))$ identifies the product (0.4) in bivariant cycle cohomology with the product (0.5) in motivic cohomology.

Proof. Set $d=\operatorname{dim}(X)$. By duality $[\mathrm{FV}, 8.2], A_{r *}\left(X, \mathbb{A}^{d}\right) \cong A_{r *}\left(\mathbb{A}^{d}, X\right) \cong A_{r *}(X)$ and

$$
A_{t *}\left(X, \mathbb{A}^{2 d}\right) \cong A_{t-d, *}\left(\mathbb{A}^{2 d}, X\right) \cong A_{t-d, *}(X) .
$$

Via these two isomorphisms, the Friedlander-Voevodsky product constructed in $[\mathrm{FV}, 8.6]$ is precisely the pairing of Corollary 4.4. 


\section{Motivic And Étale PRoduCts}

We now compare motivic and étale cohomology products. This is largely an exercise in manipulating derived categories, including Voevodsky's triangulated category $D M=D M_{-}^{e f f}(k)$, and doesn't depend upon either resolution of singularities or the choice of $\ell$.

In [V1, 3.3.1 and 3.3.3], Voevodsky constructs morphisms for each $\ell^{\nu}$ and each smooth $X$ :

$$
H_{\mathcal{M}}^{n}\left(X ; \mathbb{Z} / \ell^{\nu}(i)\right) \rightarrow H_{\text {ét }}^{n}\left(X ; \mathbb{Z} / \ell^{\nu}(i)\right)
$$

Lemma 5.2. The morphisms (5.1) are compatible with products.

Proof. Since motivic cohomology equals Zariski hypercohomology ([V1, 3.1.8 and 3.1.11], we consider the forgetful functor $\pi:(S m / k)_{\text {ét }} \rightarrow(S m / k)_{z a r}$ between sites. By [SV, 5.1], the derived direct image $\mathbb{R} \pi_{*}\left(\mu_{\ell}^{\otimes i}\right)$ and its truncation $B / \ell(i)=$ $\tau_{\leq i} \mathbb{R} \pi_{*}\left(\mu_{\ell}^{\otimes i}\right)$ are both complexes of sheaves with transfer, belonging to $D M$. Trivially, the inclusion $B / \ell(i) \rightarrow \mathbb{R} \pi_{*}\left(\mu_{\ell}^{\otimes i}\right)$ is compatible with products.

By Proposition 5.4 of $[\mathrm{SV}]$, there is a canonical morphism in $D M$ from $\mathbb{Z} / \ell(i)$ to $B / \ell(i)$. This morphism is just the $i$-fold tensor product of the canonical morphism $\mathbb{Z} / \ell(1) \rightarrow B / \ell(1)$, so it too is compatible with products. Composing gives a map from $\mathbb{Z} / \ell(i)$ to $\mathbb{R} \pi_{*}\left(\mu_{\ell}^{\otimes i}\right)$ compatible with products. Applying hypercohomology gives the map $H_{z a r}^{n}(X, \mathbb{Z} / \ell(i)) \rightarrow H_{z a r}^{n}\left(X, \mathbb{R} \pi_{*}\left(\mu_{\ell}^{\otimes i}\right)=H_{\text {ét }}^{n}\left(X, \mu_{\ell}^{\otimes i}\right)\right.$, whence the result.

Now Suslin and Voevodsky showed in [SV, 5.6 and 5.11] that the main theorem in [V2] (which requires $\ell=2$ ) implies that $\mathbb{Z} / 2(i) \rightarrow B / 2(i)$ and hence each $\mathbb{Z} / 2^{\nu}(i) \rightarrow B / 2^{\nu}(i)$ is a quasi-isomorphism of complexes of Zariski sheaves on any smooth $X$ over $k$. Since $B / 2^{\nu}(i)$ is a good truncation of $\mathbb{R} \pi_{*}\left(\mu_{\ell}^{\otimes i}\right)$, a comparison of the hypercohomology spectral sequences shows that their Zariski hypercohomology agrees in degrees at most $i$. That is, 2-primary motivic cohomology is isomorphic to étale cohomology when $0 \leq n \leq i$. Thus we deduce the following compatibility result.

Corollary 5.3. Suppose that the field $k$ admits resolution of singularities (e.g., $\operatorname{char}(k)=0)$. If $\ell=2$ and $0 \leq n \leq i$, then the products in motivic and étale cohomology are identified by Voevodsky's isomorphism

$$
H_{\mathcal{M}}^{n}\left(k ; \mathbb{Z} / \ell^{\nu}(i)\right) \stackrel{\cong}{\longrightarrow} H_{\text {ét }}^{n}\left(k ; \mathbb{Z} / \ell^{\nu}(i)\right)
$$




\section{REFERENCES}

[B1] S. Bloch, Algebraic cycles and higher K-theory, Adv. Math. 61 (1986), 267-304.

[B2] S. Bloch, The Moving Lemma for Higher Chow Groups, J. Alg. Geometry 3 (1994), 537568 .

[BL] S. Bloch and S. Lichtenbaum, A spectral sequence for motivic cohomology, Preprint (1994).

[FV] E. Friedlander and V. Voevodsky, Bivariant cycle cohomology, Preprint (1995).

[J] J. F. Jardine, Simplicial objects in a Grothendieck Topos, AMS Contemp. Math., vol. 55, 1986, pp. 193-239.

[K1] B. Kahn, Some conjectures on the algebraic K-theory of fields I: K-theory with coefficients and étale cohomology, Algebraic K-theory: Connections with Geometry and Topology (J. F. Jardine and V. P. Snaith, eds.), NATO ASI Series C, vol. 279, Kluwer, 1989, pp. 117-176.

[K2] The Quillen-Lichtenbaum Conjecture at the prime 2, Preprint (1997).

[M] J. S. Milne, Étale Cohomology, Princeton University Press, 1980.

[RW] J. Rognes and C. Weibel, Two-primary algebraic K-theory of rings of integers in number fields, preprint (1997).

[S1] A. Suslin, On the K-theory of algebraically closed fields, Invent. Math. 73 (1983), 241-245.

[S2] Higher Chow groups and étale cohomology, Preprint (1994).

[S3] _ Algebraic K-theory of fields, Proc. Intern. Congress Math., Berkeley, 1986, vol. I, Amer. Math. Soc., 1987, pp. 222-244.

[SV] A. Suslin and V. Voevodsky, The Bloch-Kato conjecture and motivic cohomology with finite coefficients, Preprint (1995).

[SV2] A. Suslin and V. Voevodsky, Relative cycles and Chow sheaves, Preprint (1994).

[V1] V. Voevodsky, Triangulated categories of motives over a field, Preprint (1995).

[V2] The Milnor Conjectures, in preparation (1996).

[W1] C. Weibel, Étale Chern classes at the prime 2, Algebraic K-theory and Algebraic Topology (P. Goerss and J. F. Jardine, eds.), NATO ASI Series C, vol. 407, Kluwer, 1993, pp. 249-286.

[W2] An introduction to homological algebra, Cambridge Univ. Press, 1994.

[W3] The 2-torsion in the K-theory of the integers, to appear, C. R. Acad. Sci. Paris (1997).

Department of Mathematics, Rutgers University, U.S.A.

E-mail address: weibel@math.rutgers.edu 